nephritis which may critically contribute to the amelioration of lupus nephritis.

\title{
A186 AUTOANTIBODY SECRETING CELLS AND LONG-LIVED PLASMA CELLS ARE ENRICHED WITHIN INFLAMED KIDNEYS OF NZB/NZW F1 LUPUS MICE AND SENSITIVE TO BORTEZOMIB TREATMENT
}

Charlotte Starke, ${ }^{1}$ Silke Frey, Ute Wellmann, ${ }^{2}$ Vilma Urbonaviciute, ${ }^{1}$ Georg Schett, ${ }^{3}$ Thomas Winkler, ${ }^{2}$ Reinhard E Voll ${ }^{1}$ Department Internal Medicine III, Nikolaus-Fiebiger Center of Molecular Medicine, Friedrich-Alexander-University Erlangen-Nuremberg, Erlangen, Germany; ${ }^{2}$ Hematopoiesis Unit, Institute for Biology, Friedrich-Alexander-University Erlangen-Nuremberg, Erlangen, Germany; ${ }^{3}$ Department of Internal Medicine 3 and Institute for Clinical Immunology, University of ErlangenNuremberg, Erlangen, Germany

\subsection{6/ard.2010.149013.29}

Background Autoantibodies to double stranded (ds)DNA represent an important marker for serological diagnosis of systemic lupus erythematosus (SLE) and may critically contribute to the pathogenesis of lupus nephritis. A long-term production of (auto-) antibodies is maintained by long-lived plasma cells. However, the anatomical sites of these cells are not yet fully identified. In contrast to short-lived plasma cells, long-lived plasma cells are extremely resistant to therapy and may often sustain refractory disease courses. Therefore, the development of novel therapeutic strategies to specifically target long-lived plasma cells is of particular importance.

Objectives Using the New Zealand black $\times$ New Zealand white (NZB/NZW) F1 mouse model of SLE the authors characterised antigen-producing cells in nephritic kidneys in respect to antigen-specificity, longevity and susceptibility to the proteasome inhibition by bortezomib (Bz).

Material and methods For discrimination between shortand long-lived plasma cells, female NZB/NZW F1 mice which already showed proteinuria and have developed high titres of anti-dsDNA antibodies were fed with 5-bromo-2'-deoxyuridine (BrdU). Short- and long-lived plasma cells within inflamed kidneys were quantified by flow cytometry. The existence of plasma cells in the kidneys was confirmed by histological analyses. Anti-dsDNA and anti-nucleolin producing cells were detected by ELISPOT assay. Diseased lupus mice were treated short-term with $\mathrm{Bz}(1 \mathrm{mg} / \mathrm{kg}$, twice in a $12 \mathrm{~h}$ interval intravenously).

Results The authors found the CD138/intracellular $\kappa / \lambda$ - $\mathrm{L}$ chain ${ }^{+}$plasma cells in renal tissue of NZB/NZW F1 mice which often formed small clusters and correlated with disease activity. Using in vivo BrdU-labelling the authors identified not only short-lived but also substantial amounts of long-lived plasma cells within nephritic kidneys. Bz treatment remarkably reduced both plasma cell compartments and T lymphocytes in inflamed kidneys. In addition, the numbers of detected cells secreting autoantibodies to dsDNA and nucleolin were significantly decreased after treatment with Bz. Moreover, the frequency of autoreactive cells in the inflamed kidneys was higher than in the spleen and bone marrow.

Conclusion Pathogenic short- and long-lived plasma cells are present in the nephritic kidneys of NZB/NZW F1 lupus mice and can be efficiently depleted by treatment with Bz. Hence, the elimination of autoreactive plasma cells from the inflamed kidneys may represent a new treatment strategy for lupus 